\title{
ON THE PEANO CURVES ASSOCIATED WITH SOME CONFORMAL MAPS
}

\author{
GERALD R. MACLANE
}

1. Salem and Zygmund [5], by use of an appropriate Taylor series with gaps, proved that there exists a function, $f(z)$, which is holomorphic in $|z|<1$, continuous in $|z| \leqq 1$, and such that the curve $w=f\left(e^{i t}\right), 0 \leqq t<2 \pi$, fills some square. Using a different type of series, Piranian, Titus, and Young [4] gave a very simple example which shows also that the curve $w=f\left(e^{i t}\right)$ can be exactly a square. The circumference $|z|=1$ is a natural boundary for the functions of Salem and Zygmund, whereas the singularities on $|z|=1$ of the function of Piranian, Titus, and Young may have measure zero. Using gap series, Schaeffer [6] has shown that $f\left(e^{i t}\right)$ can assume every value assumed by $f(z)$ in $|z|<1$. All of these proofs are essentially arithmetical, and one is led to wonder just what may be the geometry of such a startling map $w=f(z)$. The purpose of the present note is to prove a similar theorem by constructing a suitable Riemann surface onto which $|z|<1$ is mapped by $w=f(z)$. In this construction it is easy to see precisely what the corresponding Peano curve is. The resulting curve differs from the usual examples of Peano curves in that it is, in a sense, made up of linear segments.

Using this method of obtaining the desired function by first constructing a suitable Riemann surface, Ohtsuka [7] has recently proved the following theorem. ${ }^{1}$ There exists a function $w=F(z)$, bounded and analytic in $|z|<1$, such that $F\left(e^{i t}\right)=\lim _{r \rightarrow 1} F\left(r e^{i t}\right)$ has modulus 1 for almost all $e^{i t}$ on $|z|=1$, and such that, for each $c$ with $|c| \leqq 1$, the equation $F\left(e^{i t}\right)=c$ is satisfied on an uncountable set of $e^{i t}$ on $|z|=1$. The primary difference between Ohtsuka's theorem and Theorems 1 and 2 below (for the case where $D$ is the unit circle) is in the choice of the condition added to the condition that $F\left(e^{i t}\right)$ fill a domain. Namely, either $\left|F\left(e^{i t}\right)\right|=1$ for almost all $t$, or $F(z)$ is continuous in $|z| \leqq 1$. The noncountable aspect of Ohtsuka's function is similar to properties of the function of Salem and Zygmund.

2. The principal result is as follows.

Presented to the Society, October 30, 1954; received by the editors August 5, 1954 and, in revised form, October 7, 1954.

1 The author is indebted to the referee for calling attention to this paper of Ohtsuka, which appeared in print after the manuscript of the present paper was submitted. 
THEOREM 1. Let $D$ be a domain in the w-plane with the property: there exists $f(z)$, holomorphic in $|z|<1$, continuous in $|z| \leqq 1$, such that the range of values of $f(z),|z|<1$, is exactly $D$.

Then there exists $F(z)$, holomorphic in $|z|<1$, continuous in $|z| \leqq 1$, such that

$1^{\circ}$ The range of $F(z),|z|<1$, is exactly $D$.

$2^{\circ}$ The range of $F\left(e^{i t}\right), 0 \leqq t<2 \pi$, is exactly $D^{-}$.

Here $D^{-}$denotes the closure of $D$. The hypothesis on $D$ is satisfied, for instance, if $D$ is a bounded domain bounded by a finite number of disjoint Jordan curves. Namely, $f(z)$ may be taken as a function mapping $|z|<1$ onto a simply-connected covering of $D$ which covers $D$ slightly more than once.

The image of $|z| \leqq 1$ by $w=f(z)$ is compact and hence is $D^{-}$. Suppose that $\zeta=\phi(z)$ has the properties: $\phi(z)$ is holomorphic in $|z|<1$, continuous in $|z| \leqq 1$, and the range of $\phi(z),|z|<1$, is the square $Q:|\xi|<1,|\eta|<1,(\zeta=\xi+i \eta)$, while the range of $\phi\left(e^{i t}\right)$ is $Q^{-}$. Then if $Z=g(\zeta)$ maps $Q 1-1$ and conformally onto $|Z|<1$, the function $w=F(z)=f(g(\phi(z)))$ clearly has the properties stated in Theorem 1. Thus it will be sufficient to prove Theorem 1 for $D$ the square $|u|<1,|v|<1$.

Let $D_{1}, D_{2}, D_{3}, D_{4}$, denote the open quarter squares of $D, D_{i}$ in the $i$ th quadrant. Let $D_{i, j}$ denote that open quarter square of $D_{i}$ which is situated, relative to $D_{i}$, as $D_{j}$ is, relative to $D$. And so on. We shall construct a Riemann surface $S$, using each square $D, D_{i_{1}, i_{2}, \cdots, i_{p}}, 1 \leqq p$, just once. These squares are all disjoint to start with. The square $D$ is first supplied with four linear cuts running from $1 / 2+i / 2,-1 / 2$ $+i / 2,-1 / 2-i / 2$, and $1 / 2-i / 2$ to $1 / 2+i,-1 / 2+i,-1 / 2-i$, and $1 / 2-i$ respectively. The four squares $D_{j}, 1 \leqq j \leqq 4$, are supplied with corresponding cuts (one each) and attached to $D$ along these cuts. This process is now iterated: each $D_{i}$ is supplied with four cuts, similar to the four in $D$, each cut running vertically from the center of one quarter of $D_{i}$, and the four squares $D_{i, j}, 1 \leqq j \leqq 4$, are attached to $D_{i}$ along these cuts. The remaining squares are connected in an analogous fashion. This Riemann surface $S$ clearly has the properties:

(a) $S$ is simply-connected and hyperbolic.

(b) The projection of $S$ onto the w-plane is the complete open square $|u|<1,|v|<1$.

(c) $S$ may be extended to a partially bordered surface by replacing the open squares of $S$ by the corresponding closed squares. The partial border so introduced consists of various linear segments which completely cover all points of the square $|u| \leqq 1,|v| \leqq 1$ for which one 
coördinate is a dyadic rational. We have used the term partial border since $S$ also has a continuum of ideal boundary points.

Let $w=\phi(z)$ map $|z|<1 \quad 1-1$ and conformally onto $S$. This is possible because of (a). By virtue of (b), the range of $\phi(z),|z|<1$, is exactly the open square $|u|<1,|v|<1$.

By means of Carathéodory's theorem on boundary correspondence it follows readily that each segment of the partial border corresponds 1-1 and continuously with an arc of $|z|=1$. This correspondence is analytic at interior points of each segment. Assuming for the moment that $\phi(z)$ may be extended continuously throughout $|z| \leqq 1$, it follows from (c) that the range of $\phi\left(e^{i t}\right), 0 \leqq t<2 \pi$, is dense in the unit square and, being compact, must be the complete closed square $|u| \leqq 1$, $|v| \leqq 1$.

To complete the proof we must show that $\phi(z)$ may be continuously extended throughout $|z| \leqq 1$. The cut along which $D_{i_{1}, \cdots, i_{p}, i_{p+1}}$ is attached to $D_{i_{1}}, \cdots, i_{p}$ corresponds to a simple cross-cut, $C_{i_{1}}, \cdots, i_{p+1}$ of $|z|<1$ under the map $w=\phi(z)$. On $S$ the two ends of this cut are interior points of segments of the border of $S$. Thus $\phi(z)$ is holomorphic at the corresponding points of $|z|=1$ and the cross-cuts $C$ end at definite points of $|z|=1$. No two of these cross-cuts have points in common or end at a common point.

From the structure of $S$ it is clear that the cross-cuts $C$ are arranged as follows. The four cuts $C_{j}, 1 \leqq j \leqq 4$, split $|z|<1$ into five domains, one of which, $E$, corresponds to $D$. The boundary of $E$ contains all four $C_{j}$. The four remaining domains, each of which has just one $C_{j}$ on its boundary, correspond to the four parts of $S$ hung onto $D$. Inside that domain containing $C_{j}$ on its boundary lie four crosscuts ending on $|z|=1$ and not separating each other, namely $C_{j, k}$, $1 \leqq k \leqq 4$. And so on. If $E_{i_{1}}, \cdots, i_{p}$ is the domain in $|z|<1$ corresponding to $D_{i_{1}}, \ldots, i_{p}$ then every point $z_{0}$ on $|z|=1$ falls into exactly one of the two categories:

(A) $z_{0}$ is a boundary point of some $E_{i_{1}}, \cdots, i_{p}$.

(B) There exists an infinite sequence $i_{q}, q \geqq 1$, such that each $C_{i_{1}, \ldots, i q}$ separates $z_{0}$ from $E$.

In case (A), $z_{0}$ corresponds to a point of the linear border of $S$ and therefore $\phi(z)$ is continuous at $z_{0}$.

In case (B), let $G_{i_{1}}, \ldots, i q$ denote that one of the two domains in $|z|<1$ determined by $C_{i_{1}}, \ldots, i q$ which possesses $z_{0}$ as a boundary point. Since the ends of all cross-cuts $C$ are distinct, $G$ is uniquely determined and

$$
\left\{\left|z-z_{0}\right|<\epsilon\right\} \cap\{|z|<1\} \subset G_{i_{1}} \ldots . i_{q}, \quad \epsilon<\epsilon_{0}(q) .
$$


Now $G_{i_{1}}, \ldots, i q$ corresponds to a part of $S$ which lies over a square of side $2^{-q+1}$ and hence the oscillation of $\phi(z)$ in $G_{i_{1}}, \ldots, i q$ is $2^{-q+3 / 2}$. Thus it follows from (1) and the Cauchy criterion that $\phi(z)$ may be defined continuously at $z_{0}$. This completes the proof of Theorem 1 .

3. For this function $\phi(z)$ we now prove

ThEOREM 2. The singularities of $\phi(z)$ on $|z|=1$ constitute a set of measure zero.

These singularities are of two types: (1) a countable set of points corresponding to the corners of all the squares in $S$, at each of which $\phi(z)$ has an algebraic singularity (branch point of order one), and (2) points $z_{0}$ of type (B) above, which are nonalgebraic singularities of $\phi(z)$. The singularities of type (1) have measure zero, and so we examine the set, $\Sigma$, of points of type (B).

Let $S_{n}$ consist of that part of $S$ made up of squares $D, D_{1}, \cdots$, $D_{i_{1}}, \ldots, i_{n}$, i.e., all squares with no more than $n$ subscripts. The last (smallest) squares of $S_{n}$ are already supplied with the cuts along which the rest of $S$ is attached. Let the totality of these $4^{n+1}$ cuts be denoted by $\gamma_{n+1} ; \gamma_{n+1}$ is part of the boundary of $S_{n}$. Let the rest of the boundary of $S_{n}$ be $\gamma_{n+1}^{\prime}$. Under $w=\phi(z), S_{n}$ corresponds to a subdomain $H_{n}$ of $|z|<1$ which is bounded by (1) $\Gamma_{n+1}$, consisting of all cross-cuts $C_{i_{1}, \ldots, i_{n+1}}$, which corresponds to $\gamma_{n+1}$, and (2) $\Gamma_{n+1}^{\prime}$, various arcs of $|z|=1$, corresponding to $\gamma_{n+1}^{\prime}$.

Let $\omega(z)$ be the harmonic measure, in $|z|<1$, of the closed set $\Sigma$. Let $u_{n}(z)$ be the harmonic measure, in $H_{n}$, of $\Gamma_{n+1}$. Since $\Sigma$ is contained in the complement of $\Gamma_{n+1}^{\prime}$ with respect to $|z|=1, \omega(z)<u_{n}(z)$ in $H_{n}$. We shall prove that $u_{n}(z) \rightarrow 0$ as $n \rightarrow \infty$. It will then follow that $\omega(z)=0$ and hence $\Sigma$ is of measure zero. It is sufficient to show that $u_{n} \rightarrow 0$ at one fixed point, which we take to be the origin. We may assume that the map $\phi$ is normalized so that $z=0$ corresponds to $w=0$ in $D$.

To prove that $u_{n} \rightarrow 0$ we revert to $S_{n}$, where $u_{n}$ corresponds to the harmonic measure $U_{n}$, in $S_{n}$, of $\gamma_{n+1}$. Consider the two-sheeted surface $R$ made up of any one of the smallest squares in $S_{n}$ and that quarter, which lies directly underneath, of the next larger square to which the first is attached. On $R, U_{n}$ is dominated by $V_{n}$, the harmonic function on $R$ with the boundary values 1 on the four arcs of $\gamma_{n+1}$ on the boundary of $R, 1$ on the two segments where one of the two sheets of $R$ was cut from a larger square, and zero elsewhere on the boundary of $R$. Since the single arc of $\gamma_{n}$ which is contained in $R$ ends at points of the boundary of $R$ at which $V_{n}=0$, it follows that $V_{n} \leqq \delta<1$ on this arc of $\gamma_{n}$. Thus

$$
U_{n}<V_{n} \leqq \delta<1
$$

on $\boldsymbol{\gamma}_{n}$. 
These two-sheeted surfaces $R$ are similar for all $n$ and hence $\delta$ is independent of $\boldsymbol{n}$.

Comparing $U_{n-1}$ and $U_{n}$ on $S_{n-1}$,

$$
\begin{aligned}
& U_{n}<\delta=\delta U_{n-1} \\
& U_{n}=\delta U_{n-1}=0
\end{aligned}
$$

on $\gamma_{n}^{\prime}$,

and therefore

$$
U_{n}<\delta U_{n-1}
$$

on $S_{n-1}$.

At the center of $D$ we find, by iterating (2),

$$
U_{n}(0)<\delta^{n-1} U_{1}(0)<\delta^{n-1},
$$

and hence $U_{n}(0) \rightarrow 0$ as $n \rightarrow \infty$. Thus $\Sigma$ is of measure zero.

What we have proved here may also be stated as follows: the ideal boundary of $S$, as distinct from the partial border, is of harmonic measure zero.

4. The curve $\phi\left(e^{i t}\right)$ differs somewhat from the usual examples of Peano curves. The use of the squares in our construction calls to mind Hilbert's [3] example of a Peano curve. In Hilbert's example the approximating curves are free of double points and the final curve has nothing worse than quadruple points. As indicated by Hilbert, his construction may be altered slightly so that the curve has nothing worse than triple points. As proved by Hahn [2], the triple-or-worse points of such a curve must be dense in the square.

In our example each approximating curve, i.e., the boundary of $S_{n}$ less $\gamma_{n+1}$, consists entirely of multiple points. If, as before, $\Sigma$ is the set of nonalgebraic singularities of $\phi(z)$ on $|z|=1$ and $\Sigma^{\prime}$ is the complement of $\Sigma$ with respect to $|z|=1$, then the following is immediate. Let $(u, v)$ be a point of the closed unit square; then

(1) If neither $u$ nor $v$ is a dyadic rational, then $(u, v)$ corresponds to exactly one point of $\Sigma$.

(2) If exactly one of $u, v$ is a dyadic rational, then $(u, v)$ corresponds to a countable subset of $\Sigma^{\prime}$ and either one or two points of $\Sigma$, according to whether $(u, v)$ is on the boundary of the unit square or inside.

(3) If both $u$ and $v$ are dyadic rationals, then $(u, v)$ corresponds to a countable subset of $\Sigma^{\prime}$ and either one, two, or four points of $\Sigma$, according to whether $(u, v)$ is a corner point, an inner point of a side, or an interior point of the unit square.

To sum up: If neither $u$ nor $v$ is a dyadic rational then $(u, v)$ is a simple point of the curve $w=\phi\left(e^{i t}\right)$; otherwise $(u, v)$ is a point of countably infinite multiplicity.

This contrasts with the Peano curve of Salem and Zygmund where each point has multiplicity equal to the power of the continuum. 
Finally, it is not difficult to show that by altering the squares in our example into appropriate rectangles and trimming them slightly, a function $\psi$ is obtained with the same properties as those we have deduced for $\phi$, except that the curve $w=\psi\left(e^{i t}\right)$ has nothing worse than triple points. That is, Peano curves obtained from holomorphic functions need be no worse, as regards multiple points, than others.

5. If $f(z)$ is holomorphic in $|z|<1$ and its range is $G$, then clearly any limiting values of $f(z)$ on $|z|=1$ must lie in $G^{-}$. Any function $F(z)$ with the properties stated in Theorem 1 may then be described as assuming as continuous boundary values all values compatible with the restriction mentioned. This naturally calls to mind Gross' [1] entire function for which every complex number is an asymptotic value.

If $\zeta=\mu(w)$ maps the unit square onto the $\zeta$-plane cut along a ray from 0 to $\infty$, then $\mu(\phi(z))=\Phi(z)$ has the following property: $\Phi(z)$ is holomorphic in $|z|<1$, continuous in the spherical metric on $|z| \leqq 1$, and $\Phi\left(e^{i t}\right)$ assumes every complex value, including $\infty$.

6. Finally, it should be noted that our method may be used to prove more general results. For instance: let $A$ be a compact subset of $|u| \leqq 1,|v| \leqq 1$ such that (1) $A$ consists of a finite number of disjoint closed Jordan regions and a finite number of disjoint Jordan arcs, (2) $A$ is connected, and (3) $A$ contains the boundary of the unit square. Then there exists $f(z)$, holomorphic in $|z|<1$, continuous in $|z| \leqq 1$, such that the range of $f(z),|z|<1$, is exactly the open unit square and the range of $f\left(e^{i t}\right), 0 \leqq t<2 \pi$, is exactly $A$.

And of course the natural question: does there exist a function $f(z)$ with the properties stated if $A$ is an arbitrary continuous curve contained in the closed unit square and containing the boundary of the unit square?

\section{REFERENCES}

1. W. Gross, Eine ganze Funktion für die jede komplexe Zahl Konvergenzwert ist, Math. Ann. vol. 79 (1919) pp. 201-208.

2. H. Hahn, Über die Abbildung einer Strecke auf ein Quadrat, Annali di Mat. ser. III vol. 21 (1913) pp. 33-55.

3. D. Hilbert, Über die stetige Abbildung einer Linie auf ein Flächenstück, Math. Ann. vol. 38 (1891) pp. 459-460.

4. G. Piranian, C. J. Titus, and G. S. Young, Conformal mappings and Peano curves, Michigan Mathematical Journal vol. 1 (1952) pp. 69-72.

5. R. Salem and A. Zygmund, Lacunary power series and Peano curves, Duke Math. J. vol. 12 (1945) pp. 569-578.

6. A. C. Schaeffer, Power series and Peano curves, Duke Math. J. vol. 21 (1954) pp. 383-389.

7. M. Ohtsuka, Note on functions bounded and analytic in the unit circle, Proc. Amer. Math. Soc. vol. 5 (1954) pp. 533-535.

RICE INSTITUTE 